\title{
Capillary imbibition into converging tubes: Beating Washburn's law and the optimal imbibition of liquids
}

\author{
Jean-Baptiste Gorce, lan J. Hewitt, and Dominic Vella* \\ Mathematical Institute, Andrew Wiles Building, Woodstock Rd, Oxford, OX2 6GG, UK \\ E-mail: dominic.vella@maths.ox.ac.uk
}

\begin{abstract}
We consider the problem of capillary imbibition into an axisymmetric tube for which the tube radius decreases in the direction of increasing imbibition. For tubes with constant radius, imbibition is described by Washburn's law (referred to here as the 'BCLW law' to recognize the contributions of Bell, Cameron and Lucas that pre-date Washburn). We show that imbibition into tubes with a power-law relationship between radius and length generally occurs more quickly than imbibition into a constant radius tube. By a suitable choice of the shape exponent it is possible to decrease the time taken for the liquid to imbibe from one position to another by a factor of two compared to the BCLW law. We then show that a further, small, decrease in the imbibition time may be obtained by using a tube consisting of a cylinder joined to a cone of three times the cylinder length. For a given inlet radius, this composite shape attains the minimum imbibition time possible. We confirm our theoretical results with experiments on the tips of micropipettes and discuss the possible significance of these results for the control of liquid motion in microfluidic devices.
\end{abstract}




\section{Introduction}

The imbibition of a wetting liquid into a cylindrical capillary tube is one of the classic demonstrations of interfacial tension: a negative capillary pressure beneath the meniscus sucks liquid into the tube at a rate that is determined by the viscous dissipation in the liquid. In the absence of gravity (and at sufficiently early times in the presence of gravity) the relationship between the time $t$ after the start of imbibition and the penetration distance of the liquid into the medium, $z_{m}$, is diffusive, i.e.

$$
z_{m}(t) \sim t^{1 / 2}
$$

This classic result is usually referred to as Washburn's law, ${ }^{1}$ or sometimes the LucasWashburn law since Lucas obtained the same result prior to Washburn. ${ }^{2}$ However, it has recently been pointed out ${ }^{3}$ that Bell \& Cameron ${ }^{4}$ also derived the $t^{1 / 2}$ law more than a decade before either Lucas or Washburn. In view of this, and as suggested by Reyssat et al. ${ }^{3}$ we shall refer to (1) as the Bell-Cameron-Lucas-Washburn (or BCLW) imbibition law.

While the balance between surface tension and viscosity represented by the BCLW law (1) is relatively simple, in many practical applications there are complications that modify the picture significantly. For example, at very early times the BCLW law predicts that the meniscus should be moving infinitely fast. In reality, the inertia of the liquid becomes important in these early stages so that in fact the meniscus moves at a constant speed. 5,6

In other scenarios, the geometry is not that of a single, circular capillary tube. Imbibition into a porous medium may often be described by the BCLW law since the medium behaves as if the pores were cylindrical ${ }^{1,7}$ while imbibition into tubes with non-circular (but uniform) cross section exhibit the BCLW law with a modified prefactor. ${ }^{8}$ However, other geometries alter the observed scalings considerably: in media consisting of packed spheres the BCLW law is observed at early times but the meniscus subsequently advances according to some smaller power that is believed to be driven by dynamic contact angle effects. ${ }^{7,9}$ 
Even without wetting effects, the geometry of the tube may modify the observed behaviour considerably. Generally, this behaviour leads to sub-diffusive growth of the wetted portion, $z_{m} \propto t^{\beta}$ with $\beta<1 / 2$. For example, imbibition in a vertical wedge gives $\beta=1 / 3,{ }^{10,11}$ while imbibition into axisymmetric tubes with a cross-sectional radius that increases away from the opening initially follows the BCLW law before ultimately reaching a regime in which arbitrary values of $\beta<1 / 2$ can be observed by a suitable choice of tube shape. ${ }^{3}$ This slow-down of imbibition can be rationalized physically as follows: the capillary suction that drives imbibition decreases as the meniscus moves further into the tube, while the viscous dissipation resisting is still dominated by the radius of the opening at the inlet. In more complicated geometries, e.g. for tubes with sections of different radius, $z_{m}$ does not obey simple scaling laws, ${ }^{12}$ though the rate of meniscus growth is a reasonably sensitive function of the tube's cross-section. Indeed, imbibition into channels with non-uniform but axisymmetric cross sections has been used to infer the spatial variation of the channel radius. ${ }^{13,14}$

In other situations, it is desired to control the rate of imbibition. For example some reactions in microfluidic devices require control of the order in which reactants meet. ${ }^{15}$ In two-dimensional microfluidic channels, imbibition may be sped up by making one wall flexible: as the meniscus passes by, it pulls on the wall, decreasing the thickness of the channel, increasing the curvature of the meniscus and thereby driving imbibition faster. ${ }^{16,17}$ In this scenario, an effective coefficient of the BCLW law is determined and found to increase the speed of imbibition by around $40 \%$. The interaction between imbibition and elasticity has also been observed at macro-scopic scales: in 'elasto-capillary rise' the walls of the channel are completely flexible. ${ }^{11,18,19}$ Again a modification of the BCLW law was observed but because the whole device is able to deform elastically, it is found that the inlet is choked, increasing viscous dissipation and ultimately slowing down the rise compared to the rigid case in a similar manner to rigid, diverging channels. ${ }^{3}$

In this paper, we study capillary imbibition in a rigid, converging tube as shown, for 


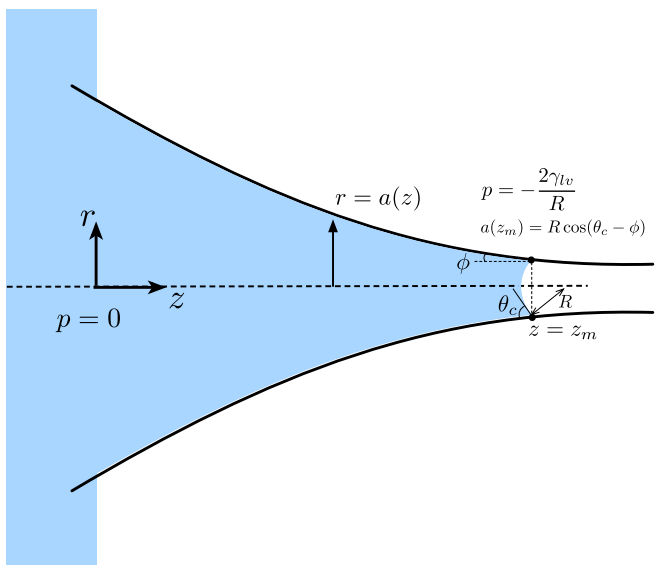

Figure 1: Imbibition into a converging capillary tube, whose radius $a(z)$ is a function of the distance from its base along the axis, $z$. Imbibition occurs from a bath of liquid at atmospheric pressure, which we take to be our pressure datum, and is driven by the capillary suction pressure beneath the meniscus. In our analysis, we neglect the role of gravity for simplicity.

example, in figure 1. Here, the magnitude of the capillary suction increases as the meniscus penetrates the tube and so, by analogy with the diverging tube, it is tempting to conclude that imbibition in such a tube will always beat the BCLW law. However, the viscous dissipation in the neighbourhood of the meniscus also increases as the meniscus approaches the tip. In some circumstances, therefore, it should be possible that capillary imbibition will, in fact, be slower than in the BCLW law. It is natural then to ask: can imbibition into a converging tube beat the BCLW law and, if so, when? We show that the answers to these questions depends critically on the tube's shape: if the tube converges too quickly then imbibition is slowed down dramatically and can even take an infinite time to imbibe to a fixed distance. However, for a wide range of tube shapes, imbibition does indeed beat the BCLW law with the time taken to travel a finite distance decreased by more than $50 \%$. We illustrate this effect with simple experiments on imbibition into glass capillary tubes and glass micro-pipette tips. 


\section{Theoretical results}

\section{General formulation}

We use lubrication theory ${ }^{20}$ to describe the velocity field of liquid during imbibition; we also neglect the role of gravity, which is valid at the small scales that are our primary motivation here. (Our use of lubrication theory requires that the tube radius varies only slowly along its axis.) As a result of this assumption, the pressure within the liquid depends only on $z$, $p=p(z)$, and the axial component of the fluid velocity, $w(r, z)$, then satisfies the reduced Stokes equation

$$
\frac{1}{r} \frac{\partial}{\partial r}\left(r \frac{\partial w}{\partial r}\right)=\frac{1}{\mu} \frac{\mathrm{d} p}{\mathrm{~d} z}
$$

where $\mu$ is the dynamic viscosity of the liquid. Eqn (2) is to be solved subject to the no-slip condition $w(a, z)=0$, which immediately gives

$$
w=\frac{1}{4 \mu} \frac{\mathrm{d} p}{\mathrm{~d} z}\left[r^{2}-a(z)^{2}\right]
$$

and hence that the fluid flux through a horizontal slice is

$$
Q(z)=2 \pi \int_{0}^{a} r w \mathrm{~d} r=-\frac{\pi[a(z)]^{4}}{8 \mu} \frac{\mathrm{d} p}{\mathrm{~d} z}
$$

However, since the boundaries of the tube are assumed to be stationary, we also have that $Q$ must be independent of $z$. Eqn (4) can therefore be rearranged to give the pressure within the liquid as a function of $z$, namely

$$
p(z)=-\frac{8 \mu Q}{\pi} \int_{0}^{z}\left[a\left(z^{\prime}\right)\right]^{-4} \mathrm{~d} z^{\prime}
$$

where we have used the assumption that the base of the tube is at atmospheric pressure, $p(z=0)=0$. The flux $Q$ is related to the speed of imbibition $\dot{z}_{m}$ by mass conservation,

$Q=\pi a\left(z_{m}\right)^{2} \dot{z}_{m}$, which may be substituted into (5) to give the pressure everywhere within 
the liquid.

To determine the (a priori unknown) rate at which the meniscus imbibes, $\dot{z}_{m}$, we use the fact that the pressure at the meniscus, $z_{m}$, must be given by the capillary suction, $-2 \gamma_{l v} / R$ where $\gamma_{l v}$ is the interfacial tension and $R$ is the radius of curvature of the meniscus. Elementary geometry, together with the assumption of a constant contact angle $\theta_{c}$, gives that $R=a\left(z_{m}\right) / \cos \left(\theta_{c}-\phi\right)$ where $\tan \phi=-a^{\prime}\left(z_{m}\right)$ is the slope of the tube wall, relative to its axis. We therefore have that the capillary suction driving imbibition is $-2 \gamma \cos \left(\theta_{c}-\phi\right) / a\left(z_{m}\right)$, which for $\phi \ll 1$ (as required by lubrication theory ${ }^{3}$ ) is $\approx-2 \gamma^{\prime} / a\left(z_{m}\right)$ with $\gamma^{\prime}=\gamma \cos \theta_{c}$. We therefore have

$$
\frac{2 \gamma^{\prime}}{a\left(z_{m}\right)}=8 \mu a\left(z_{m}\right)^{2} \dot{z}_{m} \int_{0}^{z_{m}}\left[a\left(z^{\prime}\right)\right]^{-4} \mathrm{~d} z^{\prime}
$$

or

$$
\dot{z}_{m}=\frac{\gamma^{\prime}}{4 \mu} \frac{a\left(z_{m}\right)^{-3}}{\int_{0}^{z_{m}}\left[a\left(z^{\prime}\right)\right]^{-4} \mathrm{~d} z^{\prime}} .
$$

To progress any further, it is necessary to make some explicit assumptions for the form of the radius $a(z)$.

\section{Finite cones}

To make the general formalism discussed above more concrete, we first consider the problem of capillary imbibition into a finite cone with shape equation

$$
a(z)=a_{0}\left(\frac{L-z}{L}\right)^{n}, \quad n \geq 0 .
$$

This cone has a finite width $a_{0}$ at its base and zero radius at some length $z=L$. In practice, we imagine that a very small (but finite) radius hole exists at $z=L$ allowing air to escape from the tube as the meniscus advances (this is the case in our experiments). We note also that the case $n=0$ corresponds to a tube of constant radius, for which the BCLW law holds.

With the shape equation (8), it is natural to introduce $Z_{m}=z_{m} / L$ and $T=t \times \gamma^{\prime} a_{0} /\left(\mu L^{2}\right)$, 
as dimensionless variables; (7) then becomes

$$
\dot{Z}_{m}=\left(n-\frac{1}{4}\right)\left[\left(1-Z_{m}\right)^{1-n}-\left(1-Z_{m}\right)^{3 n}\right]^{-1}
$$

This may be solved (subject to $Z_{m}(0)=0$ ) to give the meniscus position implicitly in terms of time as

$$
T=T_{\text {end }}+\frac{4}{4 n-1}\left\{\frac{\left(1-Z_{m}\right)^{3 n+1}}{3 n+1}-\frac{\left(1-Z_{m}\right)^{2-n}}{2-n}\right\}
$$

where

$$
T_{\text {end }}=\frac{4}{(2-n)(3 n+1)}
$$

is the time at which the meniscus reaches the tip of the cone, i.e. the time at which $Z_{m}=1$. (Note that $T_{\text {end }}$ only gives physically relevant values for $n<2$; we shall discuss what happens when $n \geq 2$ shortly). The solution (10) is plotted for four different values of $n$ in figure $2(\mathrm{a})$.
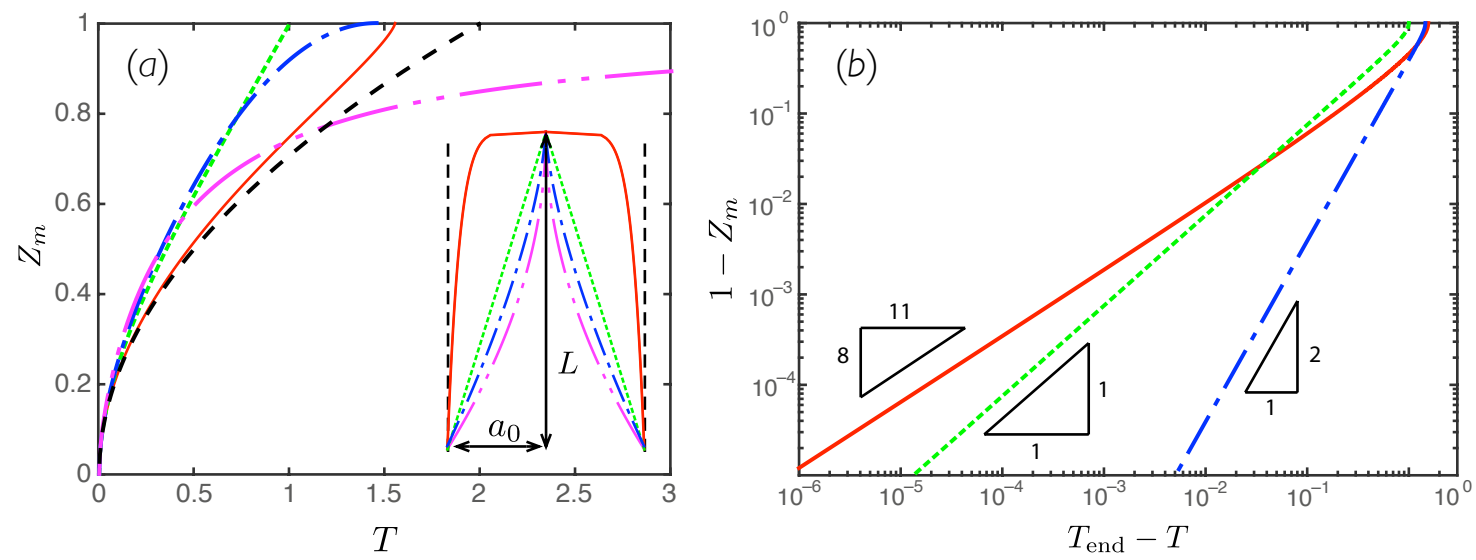

Figure 2: Analytical predictions for the imbibition of liquid into tubes with power-law radius tapering, $a(Z)=a_{0}(1-Z)^{n}$, for various values of the shape exponent $n$. (a) Main figure: The evolution of the relative meniscus position, $Z_{m}=z_{m} / L$, as a function of time $T=$ $t \times \gamma^{\prime} a_{0} /\left(\mu L^{2}\right)$ as predicted by (10) for shape exponents $n=1 / 8$ (red solid), $n=1$ (green dotted), $n=3 / 2$ (blue dash-dotted) and $n=3$ (magenta dash double-dotted) together with the BCLW imbibition law (12), (dashed curve). Inset: the shape of the corresponding tubes, using the same line styles. (b) The approach of the meniscus to the tip of the tube for the three cases with $n<2$ showing that the full analytical results do indeed recover the predicted power law behaviour, (14), as the meniscus approaches the tip. Here the different line styles are as in (a). 
From figure 2 we see that at early times the imbibition is very close to that predicted by the BCLW imbibition law, which in these dimensionless units reads

$$
Z_{m}=(T / 2)^{1 / 2}
$$

This can be derived by considering the RHS of (10) in the limit $Z_{m} \ll 1$; physically, it reflects the fact that for small meniscus penetration, the relative changes in tube radius are small so the meniscus moves as if it were imbibing into a tube of constant radius. This behaviour is generic, and has been observed in similar systems previously. ${ }^{3,13}$

We note from (11) that the dimensionless imbibition time is positive and finite, $0<$ $T_{\text {end }}<\infty$, only if $n<2$ : if $n \geq 2$, the meniscus does not reach the end of the cone within a finite time. To see this more clearly, and to uncover the motion of the meniscus as it approaches the tube tip, we let $Z_{m}=1-\zeta(T)$ and consider the dominant behaviour in (9) for $\zeta \ll 1$. We find that

$$
-\frac{\mathrm{d} \zeta}{\mathrm{d} T}= \begin{cases}\left(\frac{1}{4}-n\right) \zeta^{-3 n}, & n<1 / 4 \\ \left(n-\frac{1}{4}\right) \zeta^{n-1}, & n \geq 1 / 4\end{cases}
$$

We then have that for $\zeta \ll 1$

$$
\zeta \sim \begin{cases}\left(T_{\text {end }}-T\right)^{1 /(3 n+1)}, & n<1 / 4 \\ \left(T_{\text {end }}-T\right)^{1 /(2-n)}, & 1 / 4 \leq n<2 \\ \exp (-7 T / 4), \quad n=2 \\ \left(T-T_{*}\right)^{-1 /(n-2)}, \quad n>2,\end{cases}
$$

where the prefactors are determined by the full solution, as is the constant $T_{*}$ for $n>2$. We note that these scalings are similar to what was found recently for the motion of drops into a tapered wedge, ${ }^{21}$ though the different behaviour when $n<1 / 4$ was not noted there. 
As expected, (14) shows that for $n<2$, the meniscus reaches the tip of the cone a finite time, $T_{\text {end }}$, after the beginning of capillary imbibition. For $n=2$ it reaches the tip exponentially slowly, while for $n>2$, the meniscus only reaches the tip algebraically slowly as $T \rightarrow \infty$. This then provides an answer to our original question of which of the increased capillary suction and the increased viscous dissipation dominates as the liquid enters a narrowing tube: provided that the tube does not have too sharp a cusp $(n<2)$, the increase in capillary suction overcomes the increased dissipation and capillary imbibition proceeds quickly.

It is also of some interest to determine for which values of $n$ the meniscus reaches the tip more quickly than the BCLW imbibition (for which $T_{\text {end }}=T_{\text {end }}^{\mathrm{BCLW}}=2$ ) would. A simple calculation reveals that $T_{\text {end }}<2$ for all values of $n \in(0,5 / 3)$, with a minimum value,

$T_{\mathrm{end}}^{\min }=48 / 49$, attained at $n=n_{\mathrm{opt}}=5 / 6$. These results are shown graphically in figure 3 . Here, we emphasize two features of this result: (i) almost any degree of tapering (provided it is not too sharp at the end) will decrease the time taken to imbibe liquid to the tip when compared with imbibition into a tube of constant radius and (ii) values of $n$ close to the optimal, $n=5 / 6$, achieve imbibition times very close to the optimal one; in particular, for $n=1$ (a cone of constant angle) $T_{\text {end }}=1$, which is within $2 \%$ of the optimal value.

\section{Optimal shapes}

Having considered the specific case of power-law shaped tubes and found that the minimum imbibition time for this family of shapes occurs with $n=5 / 6$, a natural question is whether this shape is in fact optimal amongst all possible shapes? To address this question we now consider the problem of determining the tube shape $a(z)$ that minimizes the time $t_{\text {end }}$ taken for the fluid to imbibe to a given distance $L$. Since capillary imbibition naturally occurs faster in wider capillary tubes, we must somehow restrict the allowable $a(z)$ to make this problem well-posed; we choose to do so by restricting $a(z) \leq a_{0}$ everywhere, for some radius $a_{0}$. 


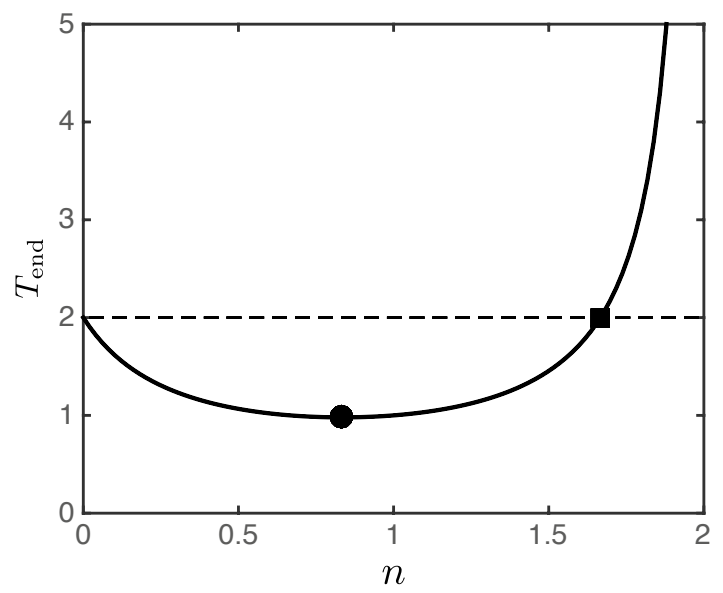

Figure 3: The dimensionless time taken for the meniscus to reach the tip of a power-law cone, $T_{\text {end }}$, as a function of the shape exponent, $n$ (solid curve). The corresponding result for a tube of constant radius $(n=0)$ is shown as the dashed line, demonstrating that capillary imbibition is 'faster' for all $n<5 / 3$, with the cross-over point $(5 / 3,2)$ indicated by a solid square. Imbibition in a power-law tube with $n=5 / 6$ reaches the tip of the tube first (i.e. has minimal $\left.T_{\text {end }}\right)$; the point $(5 / 6,48 / 49)$ is indicated by a solid circle.

Adopting the same non-dimensionalization as above, and writing $a(z)=a_{0} A(Z)$, the dimensionless time taken for the meniscus to reach $Z=1(z=L)$ is given by

$$
T_{\text {end }}=\int_{0}^{1} \frac{\mathrm{d} Z_{m}}{\dot{Z}_{m}}=4 \int_{0}^{1} A\left(Z_{m}\right)^{3} K\left(Z_{m}\right) \mathrm{d} Z_{m}
$$

where $0 \leq A(Z) \leq 1$, and

$$
K\left(Z_{m}\right)=\int_{0}^{Z_{m}}[A(Z)]^{-4} \mathrm{~d} Z
$$

A detailed calculation using the calculus of variations (given in the appendix) shows that the shape function $A(Z)$ that minimizes this time, subject to the constraint $A(Z) \leq 1$, is the piecewise linear function

$$
A(Z)= \begin{cases}1 & 0<Z<1 / 4 \\ \frac{4}{3}(1-Z) & 1 / 4<Z<1 .\end{cases}
$$

This shape is shown in the inset to figure 4, where it is compared to the fastest power-law 
shape, $A(Z)=(1-Z)^{5 / 6}$. Note that the optimal tube shape in this case consists of a constant radius cylinder attached to a linearly tapering cone with the cylinder occupying a fraction $\alpha=1 / 4$ of the total length. The corresponding imbibition time is $T_{\text {end }}=7 / 8 \approx 0.875$, which is slightly smaller than the value $48 / 49 \approx 0.980$ found for the optimal power-law tube $A(Z)=(1-Z)^{5 / 6}$.

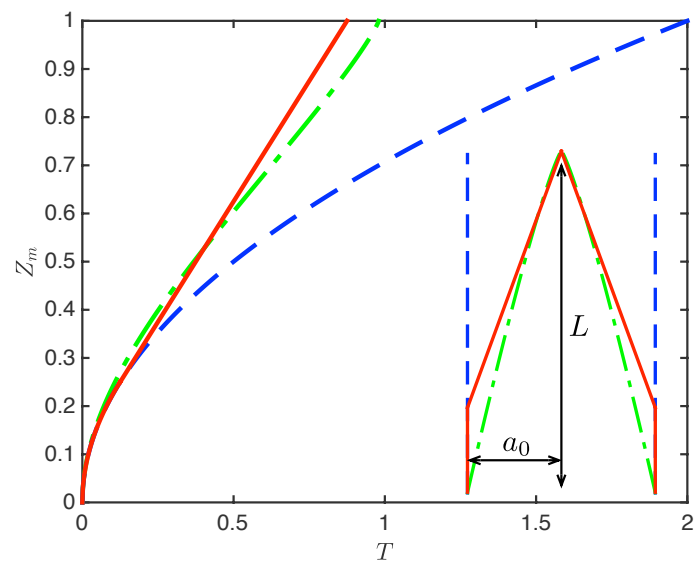

Figure 4: Main figure: The evolution of the meniscus position, $Z_{m}=z_{m} / L$, as a function of time $T=t \times \gamma^{\prime} a_{0} /\left(\mu L^{2}\right)$ for imbibition into the optimal capillary tube (17) (red solid curve), the fastest power-law tube, $A(Z)=(1-Z)^{5 / 6}$, (green dash-dotted curve) and the constant radius BCLW imbibition law (12), (blue dashed curve). Inset: The different shapes of these three tubes, with line styles as in the main figure.

A simple calculation also shows that for the optimal shape (17) imbibition precisely follows the BCLW law (12) for $T<1 / 8$ but then proceeds at constant speed, $\dot{Z}_{m}=1$, for $1 / 8<T<7 / 8$. The evolution of the meniscus position in this case is shown as the solid curve in figure 4. A comparison with the evolution obtained with the fastest power-law shape, $A(Z)=(1-Z)^{5 / 6}$, (dash-dotted curve in figure 4) shows that the meniscus in the optimal tube initially lags behind that in the fastest power-law tube but quickly overtakes it once it reaches the conical section (in particular, imbibition in the optimal tube overtakes that in an $n=5 / 6$ tube at $T \approx 0.403, Z \approx 0.528$ ). It is also perhaps of interest that the optimal shape carries a larger volume of liquid than the fastest power-law shape. 


\section{Experiments}

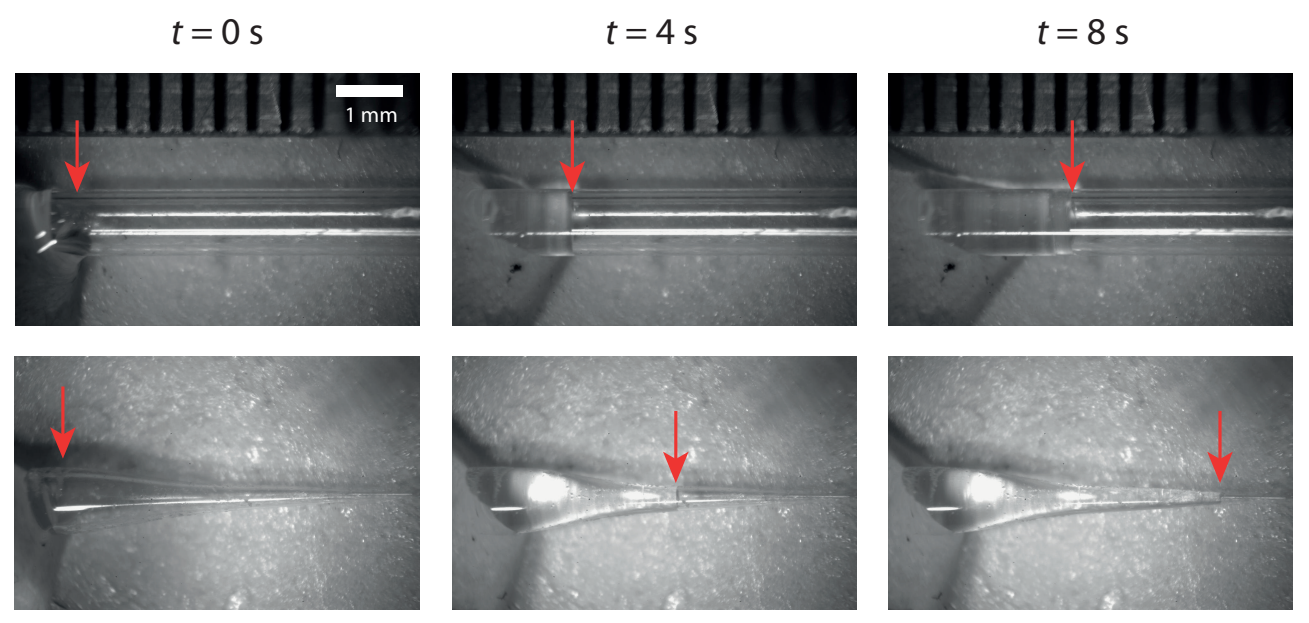

Figure 5: A capillary 'race': imbibition of glycerol into a cylindrical tube (top) and a tapered tube, a micropipette tip, (bottom) shows that capillary imbibition into converging tubes can happen faster than predicted by the BCLW law. Here both tubes have a maximal internal radius $a_{0} \approx 330 \mu \mathrm{m}$ and outer diameter of $1 \mathrm{~mm}$. Note the scale bar corresponding to $1 \mathrm{~mm}$ and that each division on the ruler corresponds to $0.5 \mathrm{~mm}$. The red arrow indicates the position of the meniscus in each image.

We tested our theoretical predictions by conducting experiments in which glycerol imbibes into the end of a micropipette tip (glycerol was used to ensure that the dynamics is dominated by viscosity). Pre-pulled micropipette tips with a base outer diameter of $1 \mathrm{~mm}$ and a tip inner diameter of $1 \mu \mathrm{m}$ were obtained commercially (FivePhoton, San Diego, CA). These tips consist of a long portion ( $L \approx 5 \mathrm{~mm}$ long) in which the inner radius is approximately constant $a_{0} \approx 330 \mu \mathrm{m}$. The tube then tapers to an inner radius $\lesssim 0.5 \mu \mathrm{m}$ over a horizontal distance of $\approx 6 \mathrm{~mm}$; as a first approximation, this tapering is approximately linear (see figure 5 for a sense of the linearity of the tapering).

We remove the portion of the tube in which the radius is constant using a diamond-tipped scribe (RS components); the experiments reported here were performed on just the tapered tip. This was then laid horizontally on a the stage of a microscope and a drop of glycerol placed at the base of the tube. (The volume of this drop, $V \gtrsim 100 \mu \mathrm{L}$, is large enough that the capillary pressure within the drop is negligible in comparison with the capillary suction that drives imbibition.) The resulting imbibition was captured at 30 frames per second 
(using a CCD camera attached to the microscope) and the resulting movie analysed using ImageJ (NIH).

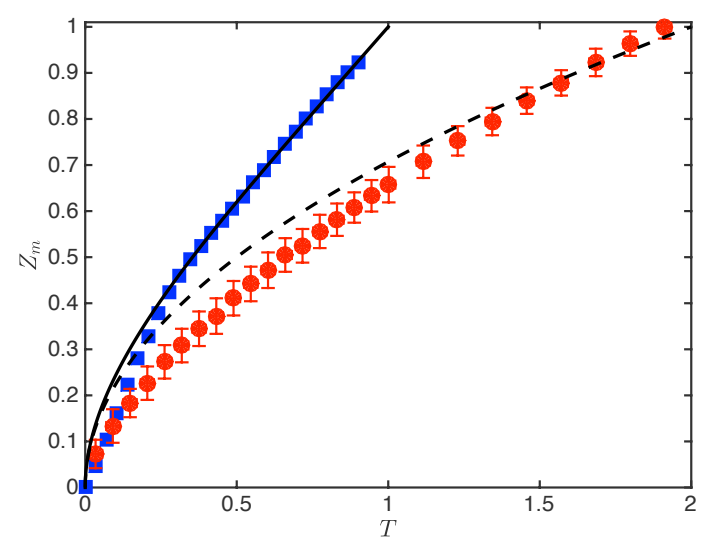

Figure 6: Experimental results for the imbibition of glycerol into a pre-pulled micropipette tip (blue squares) and a cylindrical capillary tube (red circles). The dimensionless depth of imbibition, $Z_{m}=z_{m} / L$, is shown as a function of dimensionless time $T=t \gamma^{\prime} a_{0} /\left(\mu L^{2}\right)$. For the approximately linear cone, the base diameter $a_{0} \approx 330 \mu \mathrm{m}, L \approx 6 \mathrm{~mm}$, while for the capillary tube $a_{0} \approx 513 \mu \mathrm{m}, L \approx 5 \mathrm{~mm}$. Theoretical results are shown for the imbibition into a linear cone, $n=1$, (solid curve) and into a capillary tube, $n=0$, (dashed curve). In both experiments, $\mu=1.49$ Pas and $\gamma=63 \mathrm{mN} / \mathrm{m}$; the value of the contact angle is measured from experimental images to be $\approx 75 \pm 5^{\circ}$ in each experiment. There is no parameter fitting but error bars for the constant tube experiments are obtained from two runs with the same experimental conditions.

A comparison of images from the imbibition into the tapered tube and into the constant radius portion (normally discarded) is shown in figure 5. As we expect based on the theory presented above, imbibition into a tapered cone 'beats' that into a tube of constant radius. To quantify the qualitative effect shown in figure 5, and to facilitate a quantitative comparison between theory and experiment, figure 6 shows the dimensionless meniscus position as a function of time for both cases. (For quantitative experiments with a constant radius, melting point capillary tubes of constant outer diameter $1.5 \mathrm{~mm}$ were used.) Here we see that the experimental results follow reasonably closely those predicted theoretically, though in the dimensionless terms used here the main uncertainty comes from the variation in contact angle $\theta_{c}$ between experiments and repeated measurements in the same experiment. We also see that at early times $(T \lesssim 0.2)$ the growth in both cases appears to be linear with time. 
Previous studies ${ }^{5,6}$ have shown that the inertia of the liquid causes a similar linear rise at early times, though this should ${ }^{6}$ be confined to $t \lesssim \rho a^{2} / \mu$, or $T \lesssim 10^{-5}$ in our dimensionless variables. Instead, we believe that this early-time discrepancy is caused by the pressure gradient within the feed drop of glycerol in the vicinity of the entrance to the tube - this is comparable to that within the capillary tube itself for $z_{m} \lesssim a_{0}$, but becomes negligible as the meniscus imbibes further into the tube. Nevertheless, the general behaviour predicted theoretically is consistent with that measured experimentally. In particular, the time taken for the meniscus to reach the tip of the tapered tube is roughly half the time taken for the constant radius tube, as expected. Furthermore, the fact that the tapered tube does not actually shrink to zero radius does not affect the results significantly: the additional time that the meniscus would have taken to reach the 'true' tip is a relatively small fraction $(<10 \%)$ of the total imbibition time.

\section{The two-dimensional analogue}

It is a simple matter to repeat the analysis presented earlier for the case of imbibition into

a two-dimensional channel with walls at $x= \pm a(z)$. The important changes are: (i) that the suction pressure just beneath the meniscus is now $-\gamma^{\prime} / a\left(z_{m}\right)$, rather than $-2 \gamma^{\prime} / a\left(z_{m}\right)$, because the meniscus is curved in only one direction and (ii) that the integrated conservation of mass occurs only through the thickness of the channel (rather than across the area). The two-dimensional analogue of $(7)$ is then

$$
\dot{z}_{m}=\frac{\gamma^{\prime}}{3 \mu} \frac{\left[a\left(z_{m}\right)\right]^{-2}}{\int_{0}^{z_{m}}\left[a\left(z^{\prime}\right)\right]^{-3} \mathrm{~d} z^{\prime}} .
$$

For channels with shape (8), this can be solved similarly to the axisymmetric case and leads to an expression similar to (10). Of more interest here, however, is that for $n<2$ the 
meniscus reaches the tip of the wedge at a dimensionless time

$$
T_{\mathrm{end}}^{2 D}=\frac{3}{(2-n)(1+2 n)}
$$

(The dimensionless variables here are precisely as in the axisymmetric problem.)

In this two-dimensional geometry the imbibition time for a channel with no tapering $(n=0)$ is $T_{\mathrm{end}}^{\mathrm{BCLW}}=3 / 2$, compared to $T_{\mathrm{end}}^{\mathrm{BCLW}}=2$ for a tube. Apart from this quantitative difference, the behaviour of the two-dimensional problem is very similar to that in the axisymmetric problem. In particular, the form of $T_{\text {end }}(n)$ given by $(19)$ is qualitatively very similar to that shown in figure 3 with the following quantitative differences: the minimal imbibition time, $T_{\text {end }}^{\min }=24 / 25$, is obtained with $n=n_{\text {opt }}=3 / 4$ (i.e. tapering gives a reduction in imbibition time of $36 \%$ over imbibition into a constant width channel). Furthermore, for any $0<n<3 / 2, T_{\text {end }}<T_{\text {end }}^{\mathrm{BCLW}}=3 / 2$ - for a broad range of channel shapes, tapering decreases the time taken for imbibition.

Finally, repeating the minimization of the imbibition time $T_{\text {end }}$ over all shapes (modifying the variational formulation of the problem in (20)) we find that the optimal shape is again a constant width-channel attached to a section with a constant taper to zero width at the tip, as in (17). In the 2-D case, however, the optimal channel has a constant width for a fraction $\alpha=1 / 3$ of its total length and gives an imbibition time $T_{\text {end }}=5 / 6$. Table 1 summarizes the key quantities of interest in each of the two-dimensional and axisymmetric imbibition problems.

\section{Discussion}

In this paper, we have quantified the degree of tapering that allows liquid to imbibe into a tube or channel more quickly than into a tube/channel of constant width. However, we 
Table 1: Comparison between imbibition into a tapering channel (the two-dimensional problem) and into a tapering tube (the axisymmetric problem). The optimal parameter values for imbibition into a power-law tube/channel are shown together with those for the optimal shape beyond power-law shapes (which in both axisymmetric and 2-D geometries is of the form (17) albeit with different optimal values of the cylinder-to-total length ratio, $\alpha$ ). The results for the classic BCLW imbibition are also shown for comparison.

\begin{tabular}{c|c|c|c}
\hline Problem & Quantity & 2-D channel & Axisymmetric tube \\
\hline BCLW imbibition & $T_{\text {end }}^{\mathrm{BCLW}}$ & $3 / 2$ & 2 \\
\hline Power-law tapering & $T_{\text {end }}<T_{\text {end }}^{\mathrm{BCLW}}$ for: & $0<n<3 / 2$ & $0<n<5 / 3$ \\
& $n_{\mathrm{opt}}$ & $3 / 4$ & $5 / 6$ \\
& $T_{\mathrm{end}}^{\mathrm{min}}$ & $24 / 25$ & $48 / 49$ \\
& $T_{\mathrm{end}}(n=1)$ & 1 & 1 \\
\hline Optimal tapering & $\alpha_{\mathrm{opt}}$ & $1 / 3$ & $1 / 4$ \\
& $T_{\mathrm{end}}^{\mathrm{min}}$ & $5 / 6$ & $7 / 8$ \\
\hline
\end{tabular}

have also seen that a very steep tapering $(n>2)$ prevents liquid from ever reaching the tip of the tube or channel. In each case, an optimal power-law shape exists that is close to, but not equal to, a cone of constant cone angle, i.e. $n \neq 1$; the optimal power-law shapes have an imbibition time that is roughly half (axisymmetric tube) or two-thirds (2-D channel) that in the constant width case. Allowing the optimization to be performed over all shapes with maximum radius $a_{0}$ (rather than simply for power-law shapes) we found that the truly optimal shape in both cases consists of a cylindrical tube, of length $\alpha L$ and radius $a_{0}$, connected to a linear cone of length $(1-\alpha) L$ and base $a_{0}$. This led to a small further $(10-15 \%)$ reduction in the minimum imbibition time possible with a power-law tube. Nevertheless, it is interesting that a conical tube always achieves a significant improvement in the imbibition time compared to a constant radius tube and is relatively close to the true optimum.

We note that the tapered tubes studied here are qualitatively similar to those found in some biological systems. For example, the optimal distribution of tracheids in a pine needle that minimizes the pressure drop required to transport water by transpiration has been computed. ${ }^{22}$ This optimal distribution corresponds to a tube with axially varying radius of the power-law shape used here, but with $n=1 / 4{ }^{22}$ A more closely related biological 
example is the wharf roach (Ligia exotica), which uses capillary action to move water along the outside of its legs. ${ }^{23}$ Intriguingly, the dynamics of this capillary action are such that the meniscus moves at a constant speed, $z_{m} \sim t,{ }^{23}$ similar to that seen in the optimal imbibition time problem studied here. The mechanics that lead to this motion are unclear; the geometry in of the leg of the wharf roach is extremely complicated, with micron-scale surface patterning, making any direct comparison difficult.

In technological applications, being able to move fluid as quickly as possible using surface tension may be useful in microfluidic applications, as was anticipated by van Honschoten et $a l .{ }^{16}$ and Anoop \& Sen. ${ }^{17}$ These authors suggested that including a flexible wall in a channel enhances the capillary imbibition, but that it still follows a BCLW-type $t^{1 / 2}$ scaling. However, the prefactor of the effective BCLW law is increased by up to $29 \%$ (corresponding to a filling time that is $60 \%$ of the corresponding rectangular filling time) with the precise enhancement dependent on the strength of surface tension relative to the stiffness of the flexible wall. Here we have shown that a conical channel is able to reduce the filling time by more than $50 \%$. Unlike elasticity-enhanced imbibition, however, the enhancement in the rate of imbibition due to geometry is independent of the surface tension of the liquid. We also note that combining the effects of a tapered tube with a non-circular cross-section ${ }^{8}$ might enhance imbibition since high curvature due to, for example, a groove would increase the driving suction. However, as we saw here for tubes with $n>2$, this increase in suction can be more than balanced by the concomitant increase in viscous dissipation; a more detailed study of this question is warranted.

Our results also give new insight into capillary rise between elastic elements, as considered by a number of authors. ${ }^{18,19,24}$ In particular, this 'elastocapillary rise' often induces contact between deformed objects so that the elastic objects spontaneously form the type of tapered channel considered here. ${ }^{19,24}$ The shape of the elastic elements close to the point of contact varies depending on whether the ends are 'touching' or 'sticking': ${ }^{24}$ as the meniscus advances, surface tension at first causes contact to occur between beams (which results in a wedge 
shape, $n \approx 1$ ) but then draws the elements to contact along a length (which results in an approximately cubic profile, $n \approx 3$ ). The analysis presented in this paper suggests that this difference in geometry can profoundly affect the resulting dynamics (between meniscus motion at a constant rate, for touching ends, and a meniscus that only approaches the tip asymptotically, for sticking ends). Nevertheless, the fact that elastocapillary rise may, in general, transition between different effective values of $n$ (and also that the point of contact may itself move during motion) complicates the picture somewhat, making this an interesting area for future research.

\section{Acknowledgement}

This work was partly funded by the European Research Council (ERC) under the European Union's Horizon 2020 research and innovation programme (grant agreement No 637334). We are grateful to the participants of the Oxford-Princeton Collaborative Workshop Initiative 2014 for discussions leading to this work and to Howard Stone for his subsequent comments.

\section{Appendix: The optimal imbibition problem}

The imbibition time, given in (15)-(16), may be expressed as a functional of the functions $A$ and $K$,

$$
T_{\text {end }}=\mathcal{F}[A, K] \equiv 4 \int_{0}^{1}\left[A^{3} K+\lambda\left(\frac{\mathrm{d} K}{\mathrm{~d} Z}-A^{-4}\right)\right] \mathrm{d} Z
$$

where the Lagrange multiplier $\lambda$ is introduced to enforce the relationship (16) between $A(Z)$ and $K(Z)$.

To ensure that the function extremizing (20) satisfies $0 \leq A \leq 1$, we replace $A$ in (20) with $A=\left(1-B^{2}\right)^{2}$. The Euler-Lagrange equation corresponding to variations in $B(Z)$ then 
gives that either $B=0$ (corresponding to $A(Z)=1$ ) or

$$
\lambda(Z)=-\frac{3}{4} K\left(1-B^{2}\right)^{14}
$$

The Euler-Lagrange equation corresponding to variations in $K(Z)$ then gives

$$
\frac{\mathrm{d} \lambda}{\mathrm{d} Z}=\left(1-B^{2}\right)^{6}
$$

In the case $B \neq 0, \lambda$ may be eliminated from (22) using (21). (If $B=0,(22)$ is merely an equation for $\lambda$.) Together with the constraint (16) we may then eliminate $B$ in favour of $K$ to find that

$$
\left(\frac{\mathrm{d} K}{\mathrm{~d} Z}\right)^{2}-\frac{3}{4} K \frac{\mathrm{d}^{2} K}{\mathrm{~d} Z^{2}}=0
$$

(In deriving this result, we have neglected the possibility that $1-B^{2}=0$, which corresponds to $A(Z)=0$, and hence to the maximal imbibition time). The general solution of (23) is $K(Z)=(\alpha+\beta Z)^{-3}$, which corresponds to $A(Z)=c_{1}-c_{2} Z$, where $c_{1}$ and $c_{2}$ are constants.

Our analysis thus yields two functional forms for $A(Z)$ that may correspond to optimal imbibition times, $A(Z)=1$ and $A(Z)=c_{1}-c_{2} Z$. Since $A$ must be continuous, the optimal solution must therefore take the piecewise linear form

$$
A(Z)= \begin{cases}1 & 0<Z<\alpha \\ 1-\beta \frac{Z-\alpha}{1-\alpha} & \alpha<Z<1\end{cases}
$$

for some constants $0 \leq \alpha, \beta \leq 1$. (It is easy to check that the only other option, connecting an initially linear section to a straight section $A(Z)=1$, does not yield a minimal value of $\left.T_{\text {end }}\right)$. Substituting this form for $A(Z)$ back into (15) and minimizing over $\alpha$ and $\beta$, we find that the minimum occurs for $\alpha=1 / 4, \beta=1$. This is the optimal shape given in (17). 


\section{References}

(1) Washburn, E. W. The dynamics of capillary flow. Phys. Rev. 1921, 17, 273-283.

(2) Lucas, R. Ueber das Zeitgesetz des kapillaren Aufstiegs von Flüssigkeiten. Koll. Zeit. 1918, 23, 15-22.

(3) Reyssat, M.; Courbin, L.; Reyssat, E.; Stone, H. A. Imbibition in geometries with axial variations. J. Fluid Mech. 2008, 615, 335-344.

(4) Bell, J. M.; Cameron, F. K. The flow of liquids through capillary spaces. J. Phys. Chem. 1906, 10, 658-674.

(5) Quéré, D. Inertial capillarity. Europhys. Lett. 1997, 39, 533-538.

(6) Fries, N.; Dreyer, M. The transition from inertial to viscous flow in capillary rise. $J$. Colloid Int. Sci. 2008, 327, 125-128.

(7) Lago, M.; Araujo, M. Capillary Rise in Porous Media. J. Colloid Int. Sci. 2001, 235, $35-43$.

(8) Berthier, J.; Gosselin, D.; Berthier, E. A generalization of the Lucas-Washburn-Rideal law to composite microchannels of arbitrary cross section. Microfluid Nanofluid 2015, 19, 497-507.

(9) Delker, T.; Pengra, D. B.; Wong, P.-Z. Interface Pinning and the Dynamics of Capillary Rise in Porous Media. Phys. Rev. Lett. 1996, 76, 2902-2905.

(10) Ponomarenko, A.; Quéré, D.; Clanet, C. A universal law for capillary rise in corners. J. Fluid Mech. 2011, 666, 146-154.

(11) Cambau, T.; Bico, J.; Reyssat, E. Capillary rise between flexible walls. EPL 2011, 96, 24001. 
(12) Young, W. B. Analysis of capillary flows in non-uniform cross-sectional capillaries. Colloid Surf. A 2004, 234, 123-128.

(13) Urteaga, R.; Acquaroli, L. N.; Koropecki, R. R.; Santos, A.; Alba, M.; Pallares̀, J.; Marsal, L. F.; Berli, C. L. A. Optofluidic characterization of nanoporous membranes. Langmuir 2013, 29, 2784-2789.

(14) Elizalde, E.; Urteaga, R.; Koropecki, R. R.; Berli, C. L. A. Inverse problem of capillary filling. Phys. Rev. Lett. 2014, 112, 134502.

(15) Tani, M.; Kawano, R.; Kamiya, K.; Okumura, K. Towards combinatorial mixing devices without any pumps by open-capillary channels: fundamentals and applications. Sci. Rep. 2015, 5, 10263.

(16) van Honschoten, J. W.; Escalante, M.; Tas, N. R.; Jansen, H. V.; Elwenspoek, M. Elastocapillary filling of deformable nanochannels. J. Appl. Phys. 2007, 101, 094310.

(17) Anoop, R.; Sen, A. K. Capillary flow enhancement in rectangular polymer microchannels with a deformable wall. Phys. Rev. E 2015, 92, 013024.

(18) Duprat, C.; Aristoff, J. M.; Stone, H. A. Dynamics of elastocapillary rise. J. Fluid Mech. 2011, 679, 641-654.

(19) Aristoff, J. M.; Duprat, C.; Stone, H. A. Elastocapillary imbibition. Int. J. Non- linear Mech. 2011, 46, 648-656.

(20) Batchelor, G. K. An Introduction to Fluid Dynamics; Cambridge University Press, 1967.

(21) Reyssat, E. Drops and bubbles in wedges. J. Fluid Mech. 2014, 748, 641-662.

(22) Zwieniecki, M. A.; Stone, H. A.; Leigh, A.; Boyce, C. K.; Holbrook, N. M. Hydraulic design of pine needles: one-dimensional optimization for single-vein leaves. Plant, Cell Environ. 2006, 29, 803-809. 
(23) Tani, M.; Ishii, D.; Hariyama, T.; Shimomura, M.; Okumura, K. Capillary rise on legs of a small animal and on arti cially textured surfaces mimicking them. PLOS One $\mathbf{2 0 1 4}$, 9, e96813.

(24) Taroni, M.; Vella, D. Multiple equilibria in a simple elastocapillary system. J. Fluid. Mech. 2012, 712, 273-294. 
Graphical TOC Entry

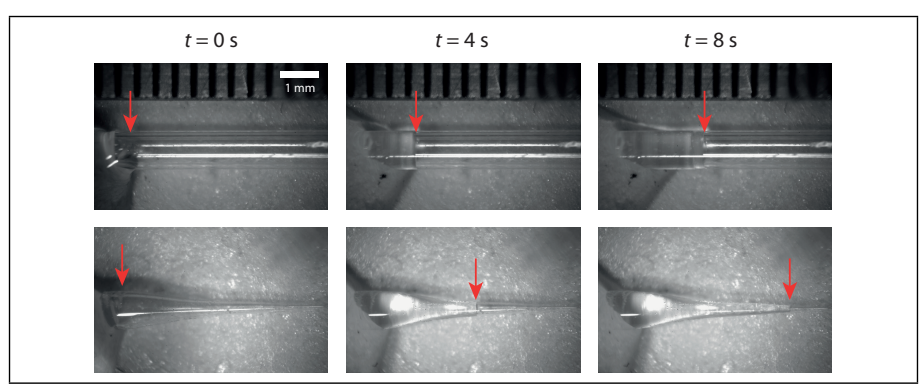

